\title{
Changes in cortical activation during mirror reading before and after training: an fMRI study of procedural learning
}

\author{
Jan Kassubek $^{\mathrm{a}, *}$, Klaus Schmidtke ${ }^{\mathrm{a}}$, Hubert Kimmig ${ }^{\mathrm{a}}$, Carl H. Lücking ${ }^{\mathrm{a}}$, \\ Mark W. Greenlee ${ }^{\mathrm{a}, \mathrm{b}}$ \\ ${ }^{a}$ Department of Neurology, University of Freiburg, Breisacher Strasse 64, 79106 Freiburg, Germany \\ ${ }^{\mathrm{b}}$ Cognitive Neuroscience, Department of Psychology, University of Oldenburg, Oldenburg, Germany
}

Accepted 13 June 2000

\begin{abstract}
The neural correlates of procedural learning were studied using functional magnetic resonance imaging (fMRI) and the mirror reading paradigm. The aim of the study was to investigate a presumed learning-related change of activation in cortical areas that are involved in the performance of a nonmotor skill. Changes in cortical blood oxygenation contrast were recorded in 10 healthy subjects while they alternatively read visually presented single mirror script words and normal script words. Responses in naive subjects were compared to those acquired after training of mirror script reading. The acquisition volume included the motor and premotor cortex, the parietal lobe and the occipital lobe including its inferior aspects. Striate and extrastriate visual areas, associative parietal cortex and the premotor cortex were bilaterally active during normal and mirror script reading. Significantly stronger activation during mirror reading was seen in BA7 and 40 (parietal associative cortex) and in BA6 (corresponding to the frontal eye fields). Simultaneous eye movement recordings indicated that activation in BA6 was related to processing components other than saccade frequency. After training, BA6 and BA7 exhibited a decrease of activation during mirror reading that significantly exceeded nonspecific changes observed in the normal script control condition. The present findings confirm the hypothesis of practice-related decrease of activation in task-related cortical areas during nonmotor procedural learning. (c) 2001 Elsevier Science B.V. All rights reserved.
\end{abstract}

Theme: Neural basis of behavior

Topic: Learning and memory: systems and functions

Keywords: Procedural learning; Mirror reading; fMRI; Parietal association cortex; Eye movement

\section{Introduction}

Procedural learning (PL) refers to the acquisition and improvement of skills through practice. It does not require conscious recollection or even awareness of the learning experience and its contents. Its results can only be shown by demonstration. Unlike declarative learning, PL is process-specific, in the sense that an acquired skill applies to all items of a given class. PL is a heterogeneous phenomenon, since there is a limitless variety of motor, perceptual and cognitive skills, and individual tasks often require more than one type of processing. Amnesic pa9588 .

*Corresponding author. Tel.: +49-761-270-5001; fax: +49-761-555-

E-mail address: kassubek@nz.ukl.uni-freiburg.de (J. Kassubek). tients have demonstrated normal learning of motor [25] and perceptual skills [12]. Learning of cognitive skills was found to be subnormal in amnesic patients, but there is evidence that additional lesions outside the hippocampal system are responsible for these deficits [32]. The observation of impaired PL in patients with degenerative basal ganglia disease suggests that motor and 'complex' prefrontostriatal loop systems play a role $[5,10,30]$.

It is assumed that PL involves the functional adaptation of neuronal structures and mechanisms that are active during the processing of a given task. A number of neuroimaging studies have investigated shifts in cerebral activation that occur in the course of sensorimotor PL, e.g., motor sequence learning [16-18]. With regard to cognitive PL, the available data are quite limited and do not allow the construction of a coherent model of the neuronal 
changes underlying this type of learning. Studies by Raichle et al. [28], van Mier et al. [39] and Sakai et al. [31] showed changes in cortical activation in the course of learning complex tasks. Although the changes described in these studies are practice-related, learning was item-specific rather than process-specific, and thus not procedural sensu stricto.

It is likely that the mechanisms underlying PL differ with the type of skill involved and with the stage of learning. PL could occur through increased efficiency of a neuronal system that is involved in a given task. It is reasonable to assume that less effortful processing is, in general, paralleled by decreased functional activation. Neuronal processing could also shift from one system to another in the course of PL, e.g., from a controlled, working-memory-dependent mode of processing to an automatic and task-specific mode that relies on associative cortex. Such a shift would be expected to lead to decrease of activation in one and increase in the other structure. For example, an increase of activation of certain motor and premotor areas has been observed in the case of motor learning [37]. Furthermore, a cortical or subcortical area could be instrumental in the implementation of a new procedure, but not itself be involved in its execution. This area would probably exhibit a temporary activation during the early stages of learning.

The aim of the present fMRI study was to investigate the changes of functional cerebral activation that occur during the acquisition of a genuinely procedural nonmotor skill. Specifically, we intended to test the hypothesis that the transition from naive to practiced performance leads to a decreased activation of those cortical areas that were involved in the processing of the skill. For this purpose, we chose the paradigm of mirror reading, a prototypic PL task that produces robust and substantial learning progress with moderate practice. Although frequently referred to as perceptual in nature, it bears an obvious cognitive component.

Several studies showed normal learning of the mirror reading skill in patients with frontostriatal lesions. This was reported in patients with frontal lobe lesions [9] and in patients with focal dorsolateral prefrontal lesions, unilateral caudate lesions, Huntington's disease and amnesia [32,33]. Although Martone et al. [24] observed a mild learning deficit in Huntington's disease patients, the cited findings are compatible with the hypothesis that PL of the mirror reading skill relies on posterior areas of the brain and does not depend on the integrity of prefrontostriatal loop systems.

To decode mirror script, untrained subjects have to perform visuospatial transformations of individual letters and combine them to words. Reading of spatially transformed vs. normal text has been shown to induce activation of the intraparietal sulcus and the superior parietal lobule in two fMRI studies $[15,27]$. Visuospatial association cortices of the parietal lobe are thus likely to be involved during unpracticed performance of the task and may be deactivated with increasing practice. A second mechanism that is probably engaged in the acquisition of the mirror reading skill is visual priming. Even when the words used in mirror reading paradigms change, the letters remain the same, and their processing may be facilitated due to repetition priming effects. Functional imaging studies have shown that repetition priming of visual material leads to a deactivation of occipital and inferior temporal visual association cortex areas that are activated during the presentation of novel stimuli [4,29].

These considerations lead to the hypothesis that mirror reading causes an activation of the parietal association cortex and the extrastriate visual association cortex in the untrained condition and a decrease of activation of these areas following PL of the skill. To test this hypothesis, we conducted an fMRI study with 10 normal subjects. In a first experiment, cortical activation induced by mirror reading and normal text reading was evaluated. In a second experiment, activations were re-evaluated after the same subjects had carried out a mirror reading training.

\section{Material and methods}

\subsection{Subjects}

The subjects were 10 healthy volunteers (six female, age range 22-32 years, mean 26 years), all of them righthanded native German speakers. None had any history of neurological or ophthalmologic disorders. All subjects had normal or corrected-to-normal visual acuity. One further subject participated on day 1 , but did not pass the performance test required for participation on day 2 . Informed consent was obtained in writing.

\subsection{Sequence of experiments}

The study consisted of two fMRI-scanning sessions in the same experimental setting on two consecutive days; on day 1 in naive condition, on day 2 after an intensive training of mirror reading. In the course of the training, which took place during several hours on day 2 prior to the evening recording session, subjects silently read 800 mirror letter words presented as lists (20 lists of 40 words each). Different word lists were used during all stages of the experiment. Before the second fMRI experiment, subjects had to pass a performance test by reading 40 eight-letter mirror script words, in order to demonstrate the successful acquisition of the skill. If the criterion of a reading time of $60 \mathrm{~s}$ (requiring an average reading time $\leq 1.5 \mathrm{~s}$ per word) was not fulfilled, the subject's data were eliminated from the study. After both fMRI experiments, the subjects' performance was controlled by a forcedchoice word recognition test involving word triplets of which only one had been shown during the scan session. 
The subjects were informed before that they would be required to recognize words presented during the scan. During the scanning sessions, the subjects did not read out loud the presented words and reaction time data were not acquired, both to avoid the activation of several additional motor and premotor areas and to minimize motion artifacts.

\section{3. fMRI activation paradigm}

On both days, the experiment consisted of three repetitions of experimental runs. Each experimental run lasted 360 s, during which a 12-slice volume was imaged repeatedly (120 volume acquisitions, $3.0 \mathrm{~s}$ each). These runs were divided into 12 epochs of $30 \mathrm{~s}$ duration, sequentially rest epochs (numbers $1,3,5,7,9,11$ ) and activation epochs (numbers 2, 6, 10: normal script; numbers 4, 8, 12: mirror script presentations). During the activation epochs, seven words were presented (rate: 1 per $4 \mathrm{~s}$, interstimulus pause: $300 \mathrm{~ms}$ ). Thus, 63 normal and mirror script words each were presented during each experiment. A model of the sequence of epochs within one run is shown as an inset in Fig. 2, middle of upper row.

During the rest (off) periods, the subjects had to fixate a white spot on neutral background produced on a screen (for details see stimulation presentation). For the mirror script stimuli, vertical mirror images of 7-8 letter German words had to be read silently from the screen. The mirror script words were flipped upside-down, but not backwards ('vertical mirror image'). In the study of Goebel et al. [15], this type of mirror image proved to be more difficult than other visual transformations. The mirror script words consisted of nouns (55\%), adjectives $(25 \%)$ and verbs (20\%). Low imaginability words were chosen to prevent changes in cerebral processing related to mental imagery once subjects had improved their mirror reading skill. An example of mirror-reversed letters is given below:

\section{spcqetap}

Normal script reading was employed as a control condition. Stimuli were German 15-25 letter words (mean 17.5), mostly abstract or technical terms, which were selected for low frequency and low imaginability $(65 \%$ composite nouns, $20 \%$ adjectives, $15 \%$ verbs). The greater length and lower frequency of normal script words was chosen to ensure attentional processing during the presentation and thus to compensate for the greater time demand involved in mirror script reading. While word length effects on the activation pattern within experiments were expected, they were not assumed to interact with learning-related changes, since changes in the neuronal processing of the overlearnt skill of normal script reading are highly unlikely.

\subsection{Stimulus presentation}

Visual stimuli were generated by a Visual Stimulus Generator graphics board and were projected onto a rearprojection screen covering the rear end of the scanner bore by an LCD projector (Panasonic), yielding a circular visual field of $30^{\circ}$ in diameter at the viewing distance of $1.2 \mathrm{~m}$. The subject lay on his/her back in the scanner, looking up into a mirror in which an image of the projection screen was reflected. The words were presented in white font on dark background. The mean luminance of individual letters was the same for both reading conditions $\left(100 \mathrm{~cd} / \mathrm{m}^{2}\right)$. Stimulus presentation was synchronized to the image acquisition procedure by means of a pulse generated by the computer controlling the scanner.

\subsection{MRI acquisition}

Imaging was performed with a $1.5 \mathrm{~T}$ whole-body Vision scanner (Siemens) equipped with a gradient system having $25 \mathrm{mT} / \mathrm{m}$ amplitude and $0.3 \mathrm{~ms}$ rise-time. The subject was positioned with his/her head in a radio-frequency receivetransmit full headcoil. Head motion was minimized with a vacuum cap and temple rests. Local variations in blood oxygenation level dependent (BOLD) response were measured using susceptibility-based functional magnetic resonance imaging, applying gradient-recalled echo-planar imaging (EPI) sequences. Twelve parallel 4-mm-thick planes, positioned slightly oblique to the axial plane and covering the parietal and occipital cortex (position of the acquisition volume see Fig. 2, left upper row), were imaged every $3 \mathrm{~s}$ using a T2*-weighted sequence (TR $3000 \mathrm{~ms}$, TE $66 \mathrm{~ms}$, flip angle $90^{\circ}, 128 \times 128$ voxels, in-plane voxel size $2 \mathrm{~mm} \times 2 \mathrm{~mm}$ ). Owing to technical limitations, the acquisition volume was limited to these 12 slices. With regard to the considerations mentioned above, the acquisition volume was positioned to include the premotor cortex, the parietal lobe and the occipital lobe, including its inferior aspects. The temporal lobe, the insular cortex and most of the prefrontal cortex were not included.

On both days, sagittal T1-weighted magnetization-prepared rapid-acquisition gradient echo (MP-RAGE) images of the entire brain (160 slices) were acquired for anatomical localization of functional responses (TR $40 \mathrm{~ms}$, TE $6 \mathrm{~ms}$, flip angle $40^{\circ}$, matrix $256 \times 256 \mathrm{~mm}^{2}$, voxel size $\left.1 \times 1 \times 1 \mathrm{~mm}^{3}\right)$. Statistical maps were transformed to the same resolution as the 3-D MR data set by interpolation. The reference points $\mathrm{AC}$ (anterior commissure) and $\mathrm{PC}$ (posterior commissure) were manually mapped, and the three reference planes AC-PC, VCA (vertical transversing AC) and midline (interhemispheric sagittal plane) were defined. Each individual brain was scaled linearly to match the Talairach atlas [36], and Talairach coordinates are reported for the center of each region of interest (ROI).

Activation of the motor cortex during mirror reading 
could be related to the control of saccadic eye movements. To relate potential changes to the saccadic activity during task performance, we monitored eye position in the MR scanner in a subgroup of subjects using our newly designed MR-Eyetracker which allows for simultaneous acquisition of eye movements and fMRI data. The system is based on infrared light reflection technique with a fiber optic system as an interface between electronic control unit and the subject's eye, for further details cf. [21]. This system has a very high temporal resolution $(2 \mathrm{kHz})$ so that it can be used to estimate eye movement velocity. That way eye movements were coregistered with fMRI in three subjects during both experimental sessions. To determine the role of the FEF in tasks requiring eye movements, we measured activation in cortical areas underlying the control of eye movements in a subsidiary fMRI control experiment. This paradigm has been described as saccadic eye movement task in a previous publication [21]. For the analysis of the eye movements all saccades were detected by a velocity threshold algorithm and were described in terms of amplitude and frequency of occurrence. We calculated the mean saccade amplitude and the frequency during the rest and activation periods, respectively (for both the saccade paradigm and the reading paradigm). The product of mean saccade amplitude and frequency of occurrence yields a measure of average saccadic activity during each single period.

\subsection{Data analysis and statistics}

The data were analyzed and visualized using BrainTools (http://www.liv.ac.uk / mariarc / mri3dX/mri3dX_download.html; cf. [34]). Residual head motion caused some image misalignment that was corrected by the 2-D motion correction program imreg (part of the AFNI package [8]). This algorithm realigns each image in the time series to the average position of that series. The motion-corrected data were then analyzed using a correlation method based on methods established by Bandettini et al. [1] and Friston et al. [13]. The time-course of the BOLD response profile is correlated with the on/off cycle of visual stimulation (i.e. the stimulus boxcar). To reduce noise, spatial smoothing of the functional signal within each slice was performed by convolution with a 2-D Gaussian function with a full width half maximum (FWHM) of $4 \mathrm{~mm}$. The time-course of each voxel was correlated with a smoothed squarewave convolved with a Gaussian (time constant $=4$ s), cf. [13]. In addition, the time-course of each voxel was smoothed with a Gaussian (time constant $=4 \mathrm{~s}$ ).

Functional activation images were constructed as pseudo-color overlays on the corresponding T1-weighted anatomical slices. Only voxels were visualized with correlation coefficients greater than 0.5 ( $P$ voxel $<0.001$ where $P$ voxel $=$ probability of a false positive, per voxel). Using the procedures of Friston et al. [13] for calculation, the effective degrees of freedom for the 120 volume acquisi- tions were approximately 34. The correlation values were then normalized to a $Z$-score statistics. Voxels are highlighted in the functional overlays whose response timecourses corresponded well with the stimulus boxcar and exceeded a $Z$-score of $3.0 \quad(P<0.001)$. Responses in selected ROIs were statistically analyzed to determine the relative magnitude of activation across different stimulus conditions. Voxel clusters containing a minimum of $4 \times 4$ contiguous voxels, all of which showed a $Z$-score of 3.0 or greater, were selected based on the pre- or post-training presence of activation within the acquisition volume. This procedure was conducted on each measurement day separately within the entire volume. Activated clusters of voxels were considered as ROIs if they were active during one or both experimental sessions. Symmetrically located clusters were identified in both hemispheres. This search was conducted in 3-D and was guided by the individual MPRAGE data set. Analysis of variance was performed on the results of BOLD responses in these ROIs. Using the BrainTools package, we interactively determined the response amplitude within ROIs located in striate, extrastriate, temporal, parietal and prefrontal cortex. The activation level was indexed by the standard deviation of the T2* signal. To weight this activation by the extent to which it is correlated with the stimulus time-course, we multiplied the response by the standardized correlation coefficient. Further details of our analysis methods have been given in previous publications [19,34]. Additional statistical analysis (ANOVA for repeated measurements) was performed on the ROI data using SPSS. We assessed the main effects of script type (normal vs. mirror-reversed), experiment (day 1 vs. day 2) and hemisphere (left vs. right), as well as the interaction script type $\times$ experiment. Calculations of the main effects of script type and experiment as well as interaction effects were performed across both hemispheres, i.e. data of corresponding areas were considered together rather than separately.

\section{Results}

\subsection{Behavioral data}

All subjects showed a significant training effect in their ability to read mirror script. In the screening test, none of them was able to read aloud 40 mirror script eight-letter words within $60 \mathrm{~s}$, whereas all of them were able to do so after the training period (the predefined criterion level required to continue the experiment).

The mean time required for 40 mirror-script words was $50.3 \mathrm{~s}$ after training (range: 40-58 s). In the word recognition test, the mean score improved significantly for mirror script words, i.e. $57 \%$ on day 2 vs. $36 \%$ on day 1 ( $P=0.001$, chi-square test). Recognition of normal script words did not change significantly (45\% vs. $53 \%$ ). These 
data confirm an improvement of subjects' ability to decode mirror script words.

\section{2. fMRI data}

\subsubsection{Main effects}

Clusters of activated voxels were identified in premotor, parietal and occipital cortical areas. The activated motor/ premotor areas corresponded to Brodmann Area (BA) 6 bilaterally, namely to the human homologs of the Frontal Eye Fields (FEF) and Supplementary Eye Field (SEF). The extrastriate cortex showed activation in BA18 and BA19, corresponding approximately to the human V2 and V3 areas, respectively. Activation was also found in the striate cortex (BA17), the human homolog of V1. In the parietal lobe, activation was found both in the superior parietal lobule (SPL), which is part of BA7, and in the inferior parietal lobe (IPL), which is part of BA40. With regard to the parietal and occipital ROIs, activation was observed on the cortical convexity rather than along the midline.

The mean Talairach coordinates of the centers of activation of each ROI are listed in Table 1. In Fig. 1A-F, the mean activation levels (in \% BOLD signal change) preand post-training in both hemispheres are given for each ROI. Fig. 2 shows slices (for $Z=48$ ) of one representative subject with the activation patterns for normal and mirror script in FEF, SEF, SPL and IPL and their changes pre- vs. post-training (together with each BOLD signal timecourse).

The mean normalized $Z$-score values of the ROIs calculated across all experimental conditions and both hemispheres (together with the numbers of subjects $N$ out of 10 showing activation in these ROIs) are listed in Table 2 .

3.2.1.1. Normal script vs. mirror script. In normal script reading versus mirror script reading in the untrained condition, a significantly different BOLD signal activation could be found both in cortical areas processing visual input (striate) and in the parietal and the premotor cortex. In the striate cortex (BA17), the BOLD signal was significantly more pronounced in normal reading $(P=$ $0.001)$. In BA18 and 19, differences between normal and mirror script reading and between hemispheres were not statistically significant. In BA6 (FEF and SEF), the BOLD signal showed a highly significant $(P<0.0001)$ increase of activation during mirror reading in comparison to normal reading. In the parietal lobe, a highly significant increase of BOLD signal during mirror reading was again detected in comparison to normal reading, both in the SPL and the IPL $(P=0.003$ and $P=0.002$, respectively $)$.

3.2.1.2. Left vs. right hemisphere. A significant hemispheric difference in cortical activation was observed in the parietal area BA40 $(P=0.0014)$ and in the prestriate BA19 $(P=0.0015)$. In all of these areas, activation was more pronounced in the left hemisphere (cf. Fig. 1). The other ROIs showed no significant differences in left vs. righthemispheric activation.

3.2.1.3. First vs. second experiment. From first to second experiment, a significant decrease in BOLD activation level was observed in the premotor BA6 $(P=0.006)$, the striate BA17 $(P=0.005)$ and the extrastriate area BA18 $(P=0.005)$. Except for the right-hemispheric BA18, this decrease was general across both script types and hemispheres (cf. Fig. 1).

\subsubsection{Procedural learning}

Effects of procedural learning of the mirror reading skill were indicated by differential changes of the activation by normal and mirror script across the two experiments, i.e. a significant statistical interaction script type $\times$ experiment. Such a significance was present in BA6 $(P=0.0025)$ and 7 $(P=0.0003)$. In these areas, the disproportional decline of activation by mirror reading was present in both hemispheres (cf. Fig. 1). In BA7, the BOLD signal remained almost unchanged during normal script reading, whereas it decreased during mirror reading. In BA40, the interaction

Table 1

Talairach coordinates of centers of activation for the regions of interest under study in left and right hemisphere ${ }^{\mathrm{a}}$

\begin{tabular}{|c|c|c|c|c|c|c|c|c|}
\hline \multirow{2}{*}{$\begin{array}{l}\text { Brodmann } \\
\text { area }\end{array}$} & \multicolumn{4}{|c|}{ Left hemisphere } & \multicolumn{4}{|c|}{ Right hemisphere } \\
\hline & $N$ & $x$ & $y$ & $z$ & $N$ & $x$ & $y$ & $z$ \\
\hline $6(\mathrm{FEF})$ & 8 & $-39.69 \pm 5.49$ & $-3.26 \pm 2.50$ & $46.82 \pm 10.18$ & 8 & $39.48 \pm 4.91$ & $-4.72 \pm 2.40$ & $49.66 \pm 9.57$ \\
\hline 7 & 7 & $-20.98 \pm 3.54$ & $-72.40 \pm 10.34$ & $38.79 \pm 6.26$ & 5 & $13.50 \pm 3.36$ & $-65.72 \pm 16.24$ & $39.12 \pm 10.44$ \\
\hline 17 & 7 & $-6.54 \pm 2.53$ & $-82.39 \pm 13.79$ & $-4.27 \pm 6.93$ & 7 & $4.60 \pm 1.50$ & $-81.39 \pm 11.92$ & $-2.18 \pm 0.61$ \\
\hline 18 & 6 & $-20.58 \pm 4.40$ & $-83.00 \pm 16.41$ & $0.85 \pm 4.43$ & 7 & $22.35 \pm 4.62$ & $-85.63 \pm 12.24$ & $-8.50 \pm 1.24$ \\
\hline 19 & 6 & $-21.90 \pm 4.49$ & $-80.59 \pm 13.68$ & $4.30 \pm 1.32$ & 6 & $21.88 \pm 5.18$ & $-77.22 \pm 13.04$ & $5.46 \pm 1.30$ \\
\hline 40 & 6 & $-37.48 \pm 1.96$ & $-65.17 \pm 3.29$ & $40.33 \pm 11.38$ & 4 & $33.70 \pm 7.33$ & $-63.30 \pm 7.35$ & $33.45 \pm 17.70$ \\
\hline $6(\mathrm{SEF})$ & 4 & $-2.85 \pm 1.68$ & $-1.67 \pm 0.52$ & $37.87 \pm 13.00$ & & & & \\
\hline
\end{tabular}

${ }^{\mathrm{a}}$ For activation levels see Table 2 .

$N=$ numbers of subjects showing significant activation.

$x$-, $y$-, $z$-values are given in $\mathrm{mm}$ and represent the mean localization of activation \pm S.E.M. within the 3-D Talairach space. 

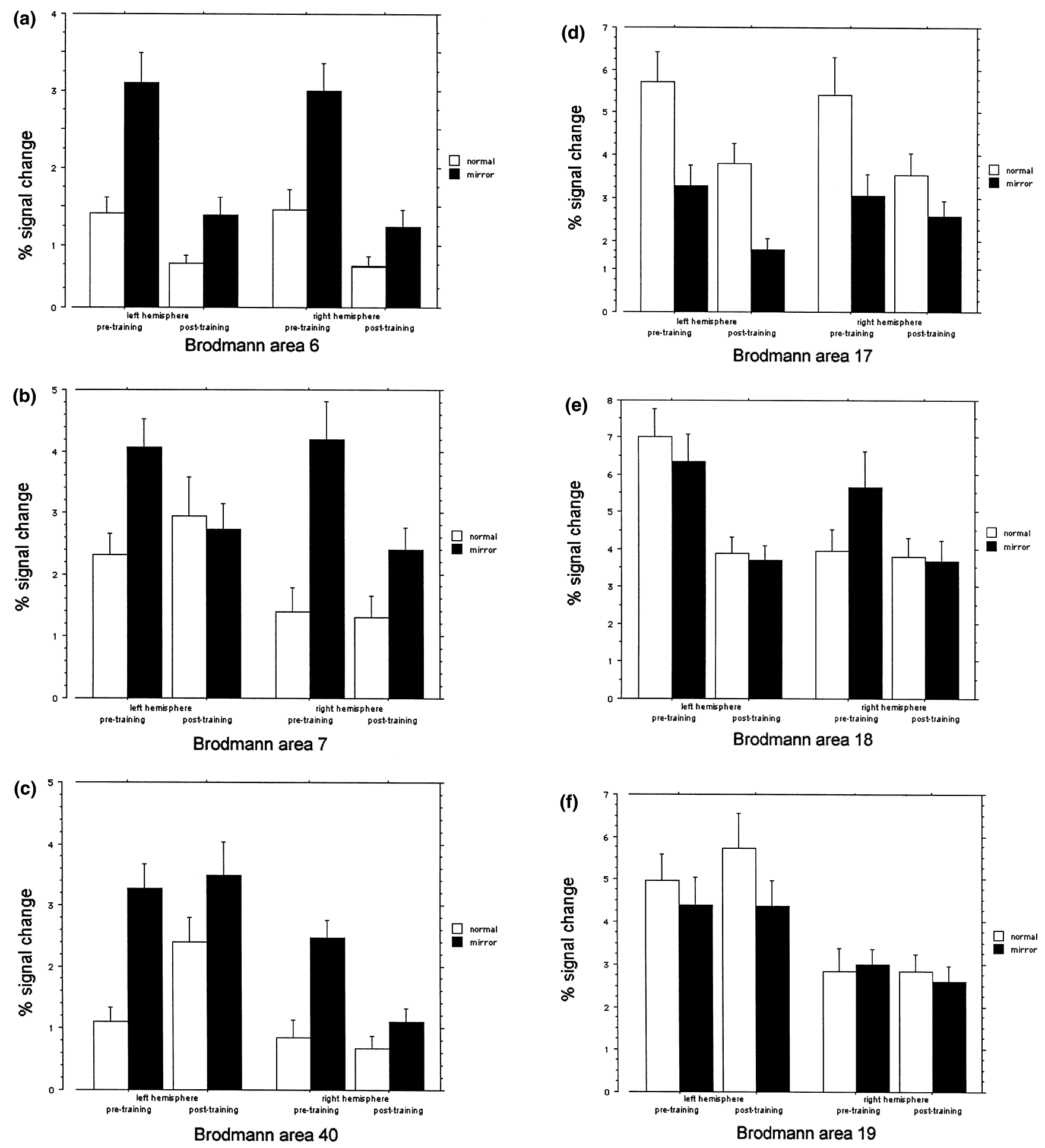

Fig. 1. Activation amplitudes (\% signal change of BOLD effect) in different ROIs for reading mirror script (black) and normal script (white) in left (LH) and right hemisphere $(\mathrm{RH})$. The left parts of the charts of each hemisphere show the activation levels pre-training, the right parts post-training. The interaction script by experiment proved to be statistically significant in BA6 $(P=0.0025)$ and BA7 $(P=0.0003)$. Error bars represent \pm S.E.M. Note the different ordinate scales.

did not reach statistical significance. Inspection of the activation charts indicated a disproportionate decrease of activation during mirror reading in the right hemisphere of two ROIs located within the extrastriate cortex. A post-hoc statistics of the right hemisphere data showed that this effect reached statistical significance (BA18: $P=0.015$, 

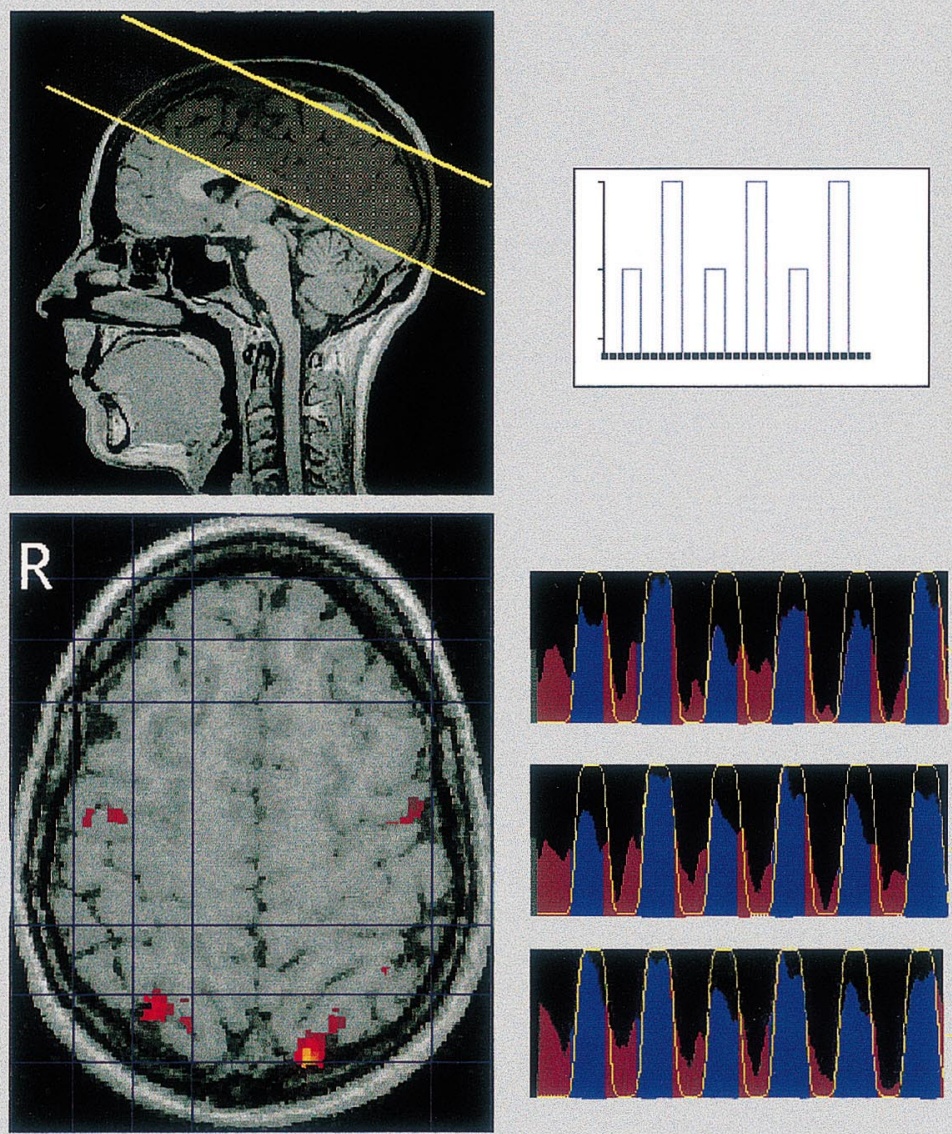

normal reading

pre-training
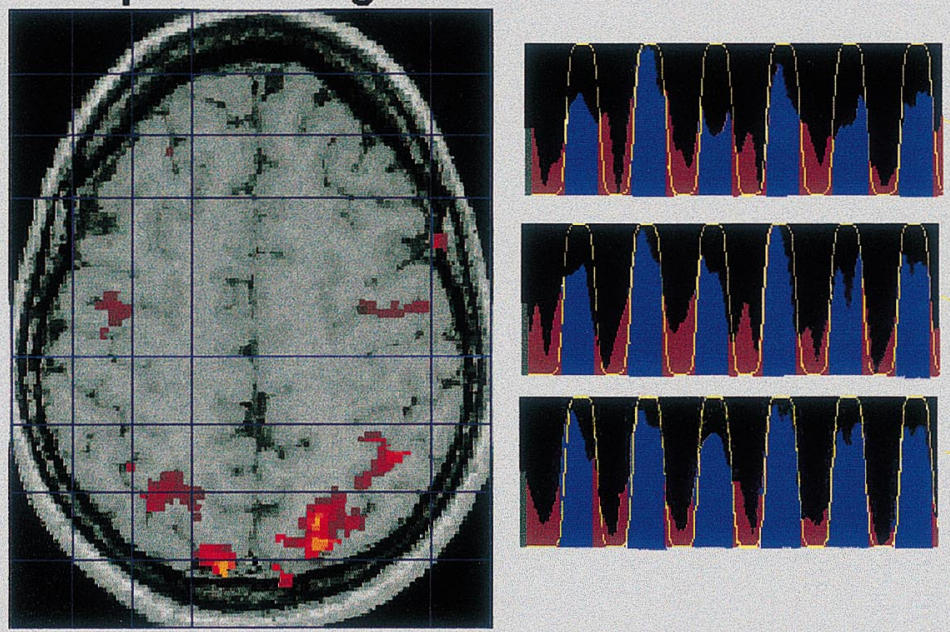

normal reading

post-training

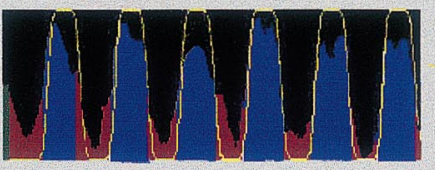

Fig. 2. Examples of BOLD results in a representative subject. In the upper row on the left the position of acquisition volume is shown, in the middle a model of the stimulus paradigm (large tracks $=$ mirror reading, small tracks=normal script reading). In the middle and lower row, left and right, representative slices of one subject (normalized to Talairach, $\mathrm{Z}=48 \mathrm{~mm}$ ) are shown demonstrating activation in BA6 (FEF/SEF), BA7 and BA40 in normal and mirror script reading pre- and post-training. An example of a 3-D visualization of activation during mirror reading pre-training is shown in the upper row on the right. BOLD time courses of the single ROIs for mirror reading are presented in the middle for pre- and post-training condition, respectively, corresponding areas are marked by arrows. $Z$-score is indexed by the color bar. Differences in activation between the conditions are visualized by different $Z$-scores and sizes of voxel clusters. 
Table 2

Mean Z-scores of activations \pm S.E.M. are shown for the different regions of interest in left and right hemisphere

\begin{tabular}{|c|c|c|c|c|c|c|c|c|c|c|}
\hline \multirow{3}{*}{$\begin{array}{l}\text { Brodmann } \\
\text { area }\end{array}$} & \multicolumn{5}{|c|}{ Left hemisphere } & \multicolumn{5}{|c|}{ Right hemisphere } \\
\hline & \multirow[t]{2}{*}{$N$} & \multicolumn{2}{|c|}{ Normal reading } & \multicolumn{2}{|c|}{ Mirror reading } & \multirow[t]{2}{*}{$N$} & \multicolumn{2}{|c|}{ Normal reading } & \multicolumn{2}{|c|}{ Mirror reading } \\
\hline & & Pre-training & Post-training & Pre-training & Post-training & & Pre-training & Post-training & Pre-training & Post-training \\
\hline $6(\mathrm{FEF})$ & 8 & $4.27 \pm 0.31$ & $3.36 \pm 0.49$ & $6.04 \pm 0.41$ & $4.58 \pm 0.43$ & 8 & $4.03 \pm 0.80$ & $3.36 \pm 0.57$ & $5.55 \pm 0.82$ & $3.81 \pm 0.62$ \\
\hline 7 & 7 & $4.50 \pm 0.80$ & $3.36 \pm 0.57$ & $6.04 \pm 0.46$ & $5.39 \pm 0.51$ & 5 & $3.98 \pm 0.69$ & $3.54 \pm 0.43$ & $5.31 \pm 0.33$ & $4.18 \pm 0.30$ \\
\hline 17 & 7 & $7.27 \pm 0.27$ & $5.89 \pm 0.63$ & $5.30 \pm 0.57$ & $4.25 \pm 0.53$ & 7 & $7.06 \pm 0.53$ & $6.15 \pm 0.64$ & $5.40 \pm 0.57$ & $4.90 \pm 0.83$ \\
\hline 18 & 6 & $7.78 \pm 0.27$ & $6.51 \pm 0.38$ & $7.58 \pm 0.28$ & $6.23 \pm 0.40$ & 7 & $6.19 \pm 0.50$ & $6.38 \pm 0.60$ & $6.55 \pm 0.86$ & $5.55 \pm 0.60$ \\
\hline 19 & 6 & $7.31 \pm 0.26$ & $6.57 \pm 0.64$ & $6.84 \pm 0.40$ & $6.13 \pm 0.68$ & 6 & $6.19 \pm 0.86$ & $5.80 \pm 0.53$ & $6.49 \pm 0.69$ & $5.31 \pm 0.57$ \\
\hline 40 & 6 & $3.71 \pm 0.72$ & $4.62 \pm 0.60$ & $5.65 \pm 0.54$ & $5.06 \pm 0.65$ & 4 & $3.17 \pm 0.52$ & $3.49 \pm 0.21$ & $4.44 \pm 0.30$ & $3.00 \pm 0.37$ \\
\hline $6(\mathrm{SEF})$ & 4 & $3.14 \pm 0.26$ & $3.14 \pm 0.33$ & $4.67 \pm 0.64$ & $4.61 \pm 0.93$ & & & & & \\
\hline
\end{tabular}

$N=$ numbers of subjects showing significant activation (only ROIs with $N \geq 4$ were considered).

BA19: $P=0.041)$. Concomitant increases of activation in any ROI were adequately checked for during the analysis, but were not observed within the acquisition volume.

\subsubsection{Analysis of FEF BOLD signal and saccadic eye} movements. Despite stronger FEF activation during mirror reading, saccade frequencies during the periods with normal letter words reading were higher than during mirror script reading periods both on day 1 (0.777 vs. 0.437 saccades/s) and on day 2 (0.716 vs. 0.324 saccades/s). The differences during mirror vs. normal script reading were significant $(P=0.013)$, whereas the differences between the pre- and post-training condition were not significant $(P=0.379)$. During the rest periods on both days only very few saccades occurred $(0.065$ and $0.032 / \mathrm{s}$, respectively). The differences of saccade frequencies between the different tasks pre- and post-training are shown in Fig. 3A. Saccade amplitudes were not different between tasks and were not altered by training.

In addition, we analyzed the amplitudes of BOLD signals in the FEF separately for the subjects with simultaneous measurements of eye movements. In the saccadic eye movement paradigm, subjects showed a significant bilateral FEF activation (Talairach coordinates within the values given in Table 1 , maximum $Z$-score 4.5) with no relevant differences both between hemispheres and between pre- and post-training experiment, cf. Fig. 3B. Note the striking contrast between lower saccadic activity and higher FEF activation during mirror reading in naive subjects.

\section{Discussion}

\subsection{Main effects of script type and hemisphere}

\subsubsection{Normal script vs. mirror script}

Brodmann areas 7, 40 and 6 displayed a significantly stronger bilateral activation during mirror reading compared to normal script reading. The reverse finding was observed in area 17. The parietal areas BA7 (convexity of the SPL) and BA40 (part of the IPL) are part of the 'dorsal pathway' and are involved in spatial processing and spatial working memory [38]. Previous functional imaging studies on mental rotation point to the SPL and IPL as important sites for this form of spatial transformation $[3,6,23,35]$. The present finding of bilateral SPL and IPL activation by mirror reading is in line with these earlier studies. In addition, few fMRI studies have specifically examined mirror reading. Goebel et al. [15] found significant lefthemispheric activation of the SPL, the posterior bank of the precentral sulcus and the occipitotemporal cortex, as well as bilateral activation along the intraparietal sulcus. Poldrack et al. [27] found bilateral activation of the posterior SPL, along the intraparietal sulcus, the occipital cortex, parts of the inferior temporal cortex, the cerebellum and the pulvinar. Dong et al. [11], who studied mirror reading of Japanese kana signs, found activation of the right SPL (plus parts of the occipital prestriate cortex, left BA6 and left BA44/45). Activation of SPL, IPL and immediately adjacent parietal cortices during untrained performance thus appears to be a reliable finding.

The primary visual cortex BA17 (V1) displayed a significantly stronger activation during normal script reading compared to mirror script reading in both hemispheres and both experiments. This finding likely reflects a word length effect, since normal script words were about twice as long as mirror script words. This difference in word lengths - designed to adjust the difficulty and time demand of the normal and mirror-script reading tasks might also explain why enhanced activation of the occipital cortex during mirror reading, as reported in previous studies [15,27], was not reproduced in our study.

The FEF could be shown to be activated during saccadic eye movements in numerous studies [e.g., 26]. In our study there is no obvious relationship between regional activation and the saccade amplitude/frequency. In fact, the normal script condition proved to be associated with greater saccadic activity compared to the mirror script condition, presumably due to the greater word lengths (Fig. 3A). We assume that the present bilateral activation of BA6 reflects a higher demand on the precision and 

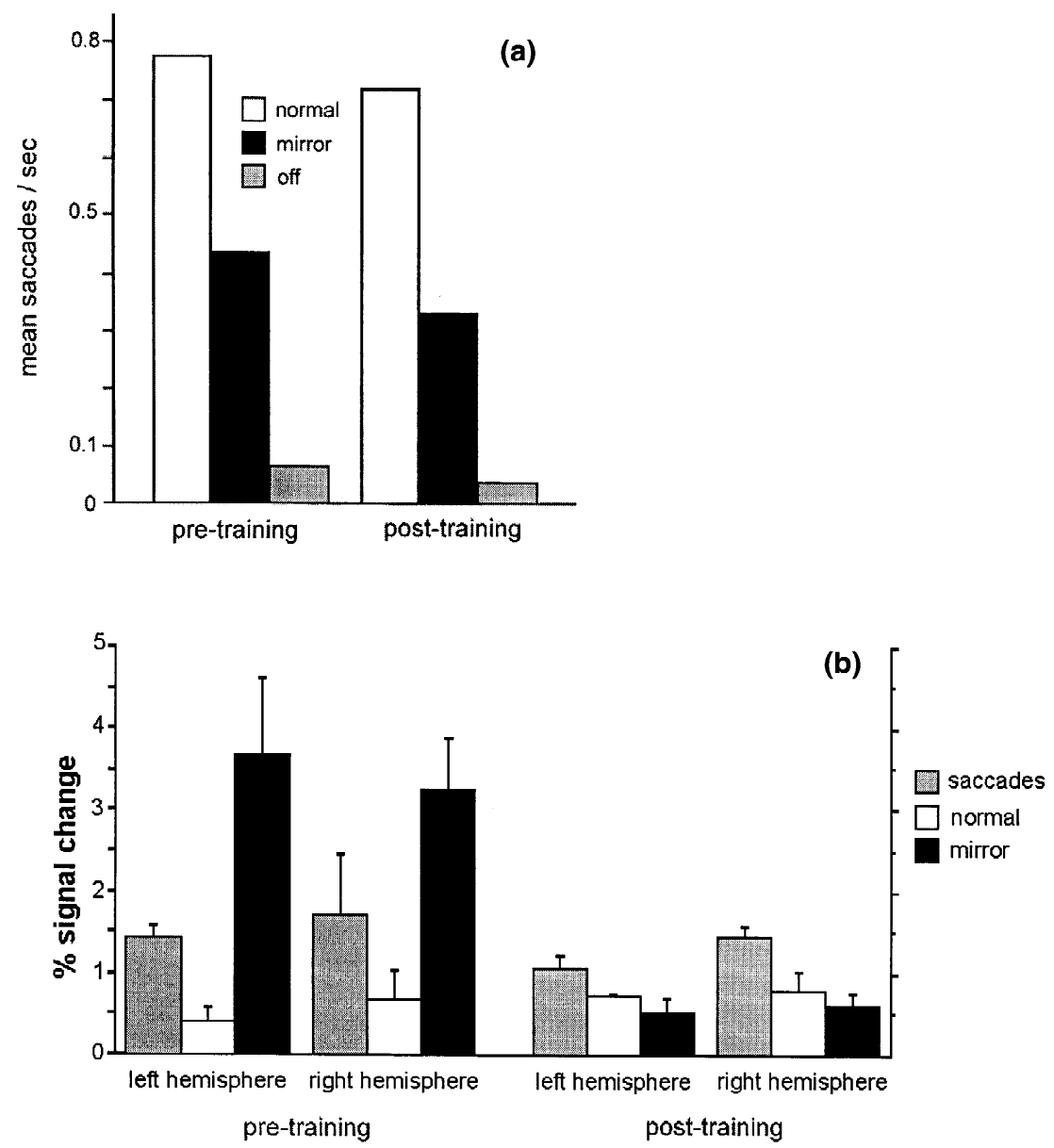

Fig. 3. (A) Saccadic activity (amplitude $\times$ frequency of saccades in $\%$ ) during normal script reading (white), mirror script reading (black) and fixation ('off', gray) in the scanning sessions. Values are presented as mean saccadic activities in the subjects whose eye movements were simultaneously measured. During fixation, there was almost no saccadic activity (as expected). Saccadic activity was markedly more pronounced during normal script reading than during mirror script reading. Values were not significantly altered pre- vs. post-training. (B) Activation amplitudes (\% signal change of BOLD effect) in prefrontal cortex (BA6, FEF) during reading mirror script (black) and normal script (white) and in the oculomotor control experiment (gray) in left (LH) and right hemisphere $(\mathrm{RH})$, as measured in the subjects whose eye movements were simultaneously recorded. In accordance with the average result of all subjects, but in contrast to saccadic activity results (cf. A), BOLD signal levels are higher during mirror reading than during normal reading and decrease markedly after training. Error bars designate \pm S.E.M.

maintenance of gaze fixation involved in mirror reading (untrained subjects have to perform mirror reading in a letter-by-letter fashion whereas the highly overlearnt skill of normal text reading relies on the recognition of entire words and groups of letters). Our findings are in agreement with several previous electrophysiological and imaging studies $[2,6,7,20,22]$, which suggest that neurons in the FEF are not only involved in the control of saccades, but also play a role in the control of fixation and possibly in directing visual attention.

\subsubsection{Left hemisphere vs. right hemisphere}

In BA19 (prestriate cortex, including visual association areas V3 and VP) and in BA40 (IPL), overall activation was significantly more pronounced in the left vs. right hemisphere. These areas are bilaterally involved in visual processing required for reading. The left hemisphere is dominant for the comprehension and processing of word content, and mirror reading is more a visuospatial than a language-related task until the transformed words are decoded.

\subsection{Procedural learning}

There is sufficient evidence for a substantial improvement in mirror reading skill between the two experiments although performance was not monitored on-line during scanning, namely the successful completion of the entry test before the second experiment, the improvement in the rate of recognition of mirror-reversed words following the second experiment, and previous findings showing a reduction of reading time by approximately $50 \%$ after a less extensive training [32]. However, it is safe to assume that subjects' mirror reading speed had not reached its asymptotic value.

In the analysis of activation differences between the pre- 
and post-training scanning sessions, potentially confounding effects have to be considered. Such effects may superimpose onto changes of cerebral activation due to learning-related modifications of task processing. Habituation in the course of increased familiarity with the general conditions of the experiment can lead to a non-specific decrease of activation. In the present study, changes of activation that are also present in the normal script condition have to be interpreted as non-specific, since normal script reading is highly overlearnt. Another possible source of bias is an interaction between learning and differences in stimulus parameters. In this study, an interaction is unlikely to occur despite the greater length of normal script words, again because normal script reading is overlearnt. Furthermore, successful procedural learning may induce secondary cognitive processing. Specifically, more successful decoding of mirror script words after training may enhance the activation of areas that are related to subvocal speech and mental imagery. However, language-related areas were not included in the acquisition volume, and the employment of low-imaginability words argues against a significant mental imagery effect. Lastly, practice effects may reduce the time required to solve a task and thus alter the 'duty cycle'. In this study, a decreased time demand for the decoding of mirror script words after training might indeed contribute to diminished activation.

The overall pattern of BOLD activation before and after training with regard to cortical topography was similar, suggesting that PL of mirror reading did not lead to a recruitment of new cortical areas within the acquisition volume. Brodmann areas 6 and 17 displayed a significant decrease of activation during the second experiment for both normal and mirror script (see Fig. 1), indicating that these changes were, at least in part, related to a nonspecific effect of habituation and reduced arousal, as discussed above. Modifications in the neuronal processing that are related to PL of the mirror reading skill were indicated by changes in the cortical activation over and above those seen for normal script. The statistical equivalent of such a change is a significant interaction of script type $\times$ experiment. A significant interaction was found for the parietal area BA7 and the premotor area BA6. Both exhibited a marked and bilateral decrease of activation in the mirror script condition, compared to less marked (BA6) or absent (BA7) changes in the normal script condition. In addition, post-hoc analysis showed a similar pattern of disproportionately stronger decreases of activation for mirror script in Brodmann areas 18 and 19 that were limited to the right hemisphere.

In the parietal association cortex, a decrease of activation in BA7 was observed during mirror reading, while the interaction did not reach significance in other ROIs including BA 40. The finding in BA7 is in line with the hypothesis that non-motor skill learning leads to a decrease in activity of cortical areas that are involved during naive performance. Specifically, the decrease of activation of
BA7 suggests an increase in the efficiency of specialized mental transformation processes which leads to a reduction in the effort and time required to decode mirror-reversed letters and to hold them in visuospatial working memory. A comparable observation was made in a preliminary report by Garavan et al. [14]. It should be borne in mind that a reduction in demand following a shift towards an 'object recognition' mode of mirror reading might play a role; a post-training increase of activation of the left fusiform gyrus was described by Poldrack et al. [27]. Such an increase was not observed in our study, which included the inferior occipital cortex (but not the inferotemporal cortex) in its acquisition volume. The decrease of activation of the extrastriate visual association areas BA18 and BA19, although unilateral, is compatible with the concept of a learning-related decrease in cortical activity due to a visual repetition priming effect. Similar decreases of activation of occipital and inferotemporal visual cortex during the repetitive presentation of similar visual stimuli have been demonstrated in previous studies $[4,29]$.

With regard to the FEF, the present substantial effect of skill learning cannot be explained by a reduction in saccadic activity, as was demonstrated by the simultaneous measurement of eye movements: after training FEF activation during mirror reading markedly decreased although saccadic activity did not significantly change pre- vs. post-training. Although these measurements were performed in only three subjects, they suggest that training had little effect on the frequency of saccadic eye movements. With reference to the interpretation of the main effect of script type in this area, it seems likely that the decrease of activation post-training is due to a decrease in the effort and precision of gaze fixation and saccadic scanning. The evaluation of FEF activation was exploratory and the interpretation of the observed decrease of activation is post-hoc. However, it is in line with the concept of PL-related cortical decreases of activation discussed above.

In summary, the findings of the present study confirm to the idea that PL of nonmotor tasks leads to a decrease of activation of cortical areas that are involved in the naive processing of the corresponding task. It is likely that more than one mechanism is involved in this functional adaptation, i.e. increased efficiency, repetition priming, and possibly decreased demand. Future studies using the mirror reading paradigm are needed to investigate a possible role of the prefrontal cortex and connected neostriatal structures. A further question is whether more extensive training leads to a pattern of functional activation that approaches the one seen in normal script reading.

\section{Acknowledgements}

We thank Thomas Mulack for help with the statistical analysis and Falk M. Krämer for help with programming. 


\section{References}

[1] P.A. Bandettini, E.C. Wong, R.S. Hinks, R.S. Tikofsky, J.S. Hyde, Time course EPI of human brain function during task activation, Magn. Reson. Med. 25 (1992) 390-397.

[2] L. Bon, C. Lucchetti, Attention-related neurons in the supplementary eye field of the macaque monkey, Exp. Brain Res. 113 (1997) $180-185$.

[3] E. Bonda, M. Petrides, S. Frey, A. Evans, Neural correlates of mental transformations of the body-in-space, Proc. Natl. Acad. Sci. USA 92 (1995) 11180-11184.

[4] R.L. Buckner, S.E. Petersen, J.G. Ojemann, F.M. Miezin, L.R. Squire, M.E. Raichle, Functional anatomical studies of explicit and implicit memory retrieval tasks, J. Neurosci. 15 (1995) 12-29.

[5] N. Butters, J. Wolfe, M. Martone, E. Granholm, L.S. Cermak, Memory disorders associated with Huntington's disease: verbal recall, verbal recognition and procedural memory, Neuropsychologia 23 (1985) 729-743.

[6] M.S. Cohen, S.M. Kosslyn, H.C. Breiter, G.J. DiGirolamo, W.L. Thompson, A.K. Anderson, S.Y. Brookheimer, B.R. Rosen, J.W. Belliveau, Changes in cortical activity during mental rotation: a mapping study using fMRI, Brain 119 (1996) 89-100.

[7] M. Corbetta, E. Akbudak, T.E. Conturo, A.Z. Snyder, J.M. Ollinger, H.A. Drury, M.R. Linenweber, S.E. Peterson, M.E. Raichle, D.C. Van Essen, G.L. Shulman, A common network of functional areas for attention and eye movements, Neuron 21 (1998) 761-773.

[8] R.W. Cox, AFNI: software for analysis and visualization of functional magnetic neuroimages, Comput. Biomed. Res. 29 (1996) $162-173$.

[9] I. Daum, H. Ackermann, M.M. Schugens, C. Reimold, J. Dichgans, N. Birbaumer, The cerebellum and cognitive functions in humans, Behav. Neurosci. 107 (1993) 411-419.

[10] M.R. DeLong, A.P. Georgopoulos, M.D. Crutcher, Cortico-basal ganglia relations and coding of motor performance, Exp. Brain Res. 49 (Suppl. 7) (1983) 30-40.

[11] Y. Dong, H. Fukuyama, M. Honda, T. Okada, T. Hanakawa, J. Konishi, H. Shibasaki, Essential role of the right superior parietal cortex in Japanese mirror reading: an fMRI study [Abstract], NeuroImage 9 (1999) S383.

[12] J. Ewert, H.S. Levin, M.G. Watson, Z. Kalisky, Procedural memory during posttraumatic amnesia in survivors of severe closed head injury. Implications for rehabilitation., Arch. Neurol. 46 (1989) 911-916.

[13] K.J. Friston, A.P. Holmes, J.B. Poline, P.J. Grasby, S.C. Williams, R.S. Frackowiak, R. Turner, Analysis of fMRI time-series revisited, NeuroImage 2 (1995) 45-53.

[14] H. Garavan, D. Kelly, A. Rosen, A.S. Bloom, S.M. Rao, E.A. Stein, Practice effects on functional activation in a working memory task [Abstract], NeuroImage 9 (1999) S938.

[15] R. Goebel, D.E.J. Linden, H. Lanfermann, F.E. Zanella, W. Singer, Functional imaging of mirror and inverse reading reveals separate coactivated networks for oculomotion and spatial transformations, NeuroReport 9 (1998) 713-719.

[16] I.H. Jenkins, D.J. Brooks, P.D. Nixon, R.S. Frackowiak, R.E. Passingham, Motor sequence learning: a study with positron emission tomography, J. Neurosci. 14 (1994) 3775-3790.

[17] M. Jueptner, C.D. Frith, D.J. Brooks, R.S. Frackowiak, R.E. Passingham, Anatomy of motor learning. II. Subcortical structures and learning by trial and error, J. Neurophysiol. 77 (1997) 13251337.

[18] A. Karni, G. Meyer, P. Jezzard, M.M. Adams, R. Turner, L.G. Ungerleider, Functional MRI evidence for adult motor cortex plasticity during motor skill learning, Nature 377 (1995) 155-158.

[19] J. Kassubek, M. Otte, T. Wolter, M.W. Greenlee, T. Mergner, C.H.
Lücking, Brain imaging in a patient with hemimicropsia, Neuropsychologia 37 (1999) 1327-1334.

[20] S. Kastner, M.A. Pinsk, P. De Weerd, R. Desimone, L.G. Ungerleider, Increased activity in human visual cortex during directed attention in the absence of visual stimulation, Neuron 22 (1999) 751-761.

[21] H. Kimmig, M.W. Greenlee, F. Huethe, T. Mergner, MR-Eyetracker: a new method for eye movement recording in fMRI, Exp. Brain Res. 126 (1999) 443-449.

[22] Y. Kodaka, A. Mikami, K. Kubota, Neuronal activity in the frontal eye field of the monkey is modulated while attention is focused on to a stimulus in the peripheral visual field, irrespective of eye movement, Neurosci. Res. 28 (1997) 291-298.

[23] S.M. Kosslyn, N.M. Alpert, W.L. Thompson, C.F. Chabris, S.L. Rauch, A.K. Anderson, Identifying objects seen from different viewpoints: a PET investigation, Brain 117 (1994) 1055-1071.

[24] M. Martone, N. Butters, M. Payne, J.T. Becker, S.S. Sax, Dissociation between skill learning and verbal recognition in amnesia and dementia, Arch. Neurol. 41 (1984) 965-970.

[25] B. Milner, Les troubles de la memoire accompagnant des lesions hippocampiques bilaterales, in: P.M. Milner, S. Glickman (Eds.), Physiologie de l'Hippocampe, Centre Nationale de la Recherche Scientifique, 1962, pp. 257-270.

[26] T. Paus, S. Marrett, K.J. Worsley, A.C. Evans, Extraretinal modulation of cerebral blood flow in the human visual cortex: implications for saccadic suppression, J. Neurophysiol. 74 (1995) 2179-2183.

[27] R.A. Poldrack, J.E. Desmond, G.H. Glover, J.D.E. Gabrieli, The neural basis of visual skill learning: an fMRI study of mirror reading, Cerebr. Cort. 8 (1998) 1-10.

[28] M.E. Raichle, J.A. Fiez, T.O. Videen, A.M. MacLeod, J.V. Pardo, P.T. Fox, S.E. Petersen, Practice-related changes in human brain functional anatomy during nonmotor learning, Cerebr. Cort. 4 (1994) 8-26.

[29] P.J. Reber, C.E. Stark, L.R. Squire, Cortical areas supporting category learning identified using functional MRI, Proc. Natl. Acad. Sci. USA 95 (1998) 747-750.

[30] J.A. Saint-Cyr, A.E. Taylor, A.E. Lang, Procedural learning and neostriatal dysfunction in man, Brain 111 (1988) 941-959.

[31] K. Sakai, O. Hikosaka, S. Miyauchi, R. Takino, Y. Sasaki, B. Putz, Transition of brain activation from frontal to parietal areas in visuomotor sequence learning, J. Neurosci. 18 (1998) 1827-1840.

[32] K. Schmidtke, R. Handschu, H. Vollmer, Cognitive procedural learning in amnesia, Brain and Cognition 32 (1996) 441-467.

[33] K. Schmidtke, H. Manner, H. Vollmer, Deficits of procedural learning in focal prefronto-striatal lesions and Huntington's disease [Abstract], J. Neurol. 245 (1998) 354.

[34] A.T. Smith, M.W. Greenlee, K.D. Singh, F.M. Krämer, J. Hennig, The processing of first- and second-order motion in human visual cortex assessed by fMRI, J. Neurosci. 18 (1998) 3816-3830.

[35] G.A. Tagaris, S.G. Kim, J.P. Strupp, P. Andersen, K. Ugurbil, P. Georgopoulos, Quantitative relations between parietal activation and performance in mental rotation, NeuroReport 7 (1996) 773-776.

[36] J. Talairach, P. Tournoux (Eds.), Co-Planar Atlas of the Human Brain: 3-Dimensional Proportional System, Thieme, Stuttgart, New York, 1988.

[37] L.G. Ungerleider, Functional brain imaging studies of cortical mechanisms for memory, Science 270 (1995) 769-775.

[38] L.G. Ungerleider, S.M. Courtney, J.V. Haxby, A neural system for human visual working memory, Proc. Natl. Acad. Sci. USA 95 (1998) 883-889.

[39] H. Van Mier, L.W. Tempel, J.S. Perlmutter, M.E. Raichle, S.E. Petersen, Changes in brain activity during motor learning measured with PET: effects of hand of performance and practice, J. Neurophysiol. 80 (1998) 2177-2199. 